EISSN: 2706 -7947 ISSN: 2077- 4613

DOI: 10.36632/mejas/2021.11.2.44

Journal homepage: www.curresweb.com

Pages: 570-578

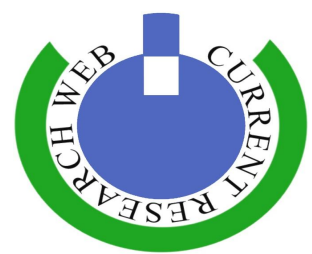

\title{
Reducing Some Abiotic Stress Factors Effect on Valencia Orange Tree Growth and Productivity under Middle Egypt Conditions
}

\author{
Randa E.Y. Habasy, Huda M.H. Ismaiel and Abd Al Rahman M. Abd Al Rahman
}

Citric Res. Dept., Hort. Res. Instit., Agric. Res. Center, Giza, Egypt

\author{
Received: 20 April $2021 \quad$ Accepted: 10 June $2021 \quad$ Published: 25 June 2021
}

\begin{abstract}
This study was conducted during 2018 and 2019 seasons, to elucidate the effect of single and combined applications of glutathione, ascorbic acid and citric acid each at three different concentrations; 250,500 and $1000 \mathrm{ppm}$ on growth characters, nutritional status, yield, physical and chemical characteristics of Valencia orange trees. No doubt single applications of glutathione, ascorbic and citric acid improved the most of parameters under study. But the combined of each at high concentration (1000 ppm) were very effective in stimulating growth characteristics and nutritional status of the trees and improving yield and fruit quality of Valencia orange trees relative to the check treatment. Using these compounds together was more effective than using each alone. Therefore, to improve the yield and fruit quality of Valencia orange grown under Bani- Suef conditions, it is recommended to foliar spray the trees three times, just after fruit setting (the $1^{\text {st }}$ week of May) and two months, intervals (the $1^{\text {st }}$ week of July and September) with a mixture containing glutathione, ascorbic acid and citric acid each at $1000 \mathrm{ppm}$.
\end{abstract}

Keywords: glutathione, ascorbic acid, citric acid, Valencia orange.

\section{Introduction}

Citrus is suggested to be one of the most important cash crops all over the world. It is occupying the $1^{\text {st }}$ rank among economic fruit crops in Egypt and essential component of some human nutritional requirements such as vitamins, minerals and organic acid ...etc. Valencia orange is considered one of oranges cultivars that its production was developed in a high rate during the last years in Egypt. Valencia orange fruits take the lead in the Egyptian exportation to foreign markets. However, poor cropping is considered to be a serious and major problem that faces Valencia orange grown in Bani Suif region (which suffer from high temperature and low of relative humidity) that lead to extensive dropping of the flowers and fruits and poor fruit setting. To reduce the harmful effect of this conditions the major cultural practices such as: improving tree growth, nutritional status, fruit retention, yield and fruit quality some antioxidants components must be applied.

Antioxidants compounds have auxine action and have synergistic effect on growth and productivity of most fruit trees. So, it's considered the most appropriates use on the fruit trees under field conditions and is favourable possibility. In this concern, further and additional studies are needed to elucidate their mode of action on fruit trees and to find cheap antioxidants that are beneficial for enhancing growth and productivity. At the same time they are safe to reduce of the cell senescence. Whereas, it's important to protect the cell from senescence enhance the cell division and the biosynthesis of organic foods and control the incidence of fungal attack (Raskin, 1992). The positive action of the antioxidants in chelating these radicals could result in extending the shelf life of plant cells and producing vigorous plants (Vianello and Marci, 1991; Sandermann et al., 1998; Klessig et al., 2000; Rao et al., 2000).

Vitamins such as citric and ascorbic acids act as co-enzymes in a number of enzymes systems, thus, they take apart in the regulation of metabolism. Recently, it was suggested that vitamins participate in plant growth and development indirectly by enhancing the endogenous levels of various growth

Corresponding Author: Huda M.H. Ismaiel, Citric Res. Dept., Hort. Res. Instit., Agric. Res. Center, Giza, Egypt. E-mail: huda_sps122@hotmail.com 
factors such as cytokinins and gibberellins. Most vitamins are synthesized in leaves and trans-located in the problem. Vitamins with their antioxidative properties play an important role in plant defense against oxidative stress induced by surfactants and selected pesticides. Application of vitamins is accompanied with enhancing alpha keto glutaric acid biosynthesis, which is combining with amines to form amino acids and proteins (Orth et al., 1993).

Glutathione (cysteine + glycine + glutamic acid) is the most important non protein thiol present in plants. It is essential in sulfur metabolism and defense against most stresses. It is important pool of reduced surface and it regulates sulfur uptake at root level. Reduced glutathione the major water soluble antioxidant in photosynthetic and non- photosynthetic tissues, reacting directly or indirectly with reactive oxygen species, contribute to maintain the integrity of cell structure and proper functions of various metabolic pathways. In addition, its effects on expression of defense genes glutathione may also be involved in redox control of cell division and enhancing growth of the plants (Mulleineaux and Rausch, 2005). The results of (Abd Elaal et al., (2012); Gad El-Kareem, (2012); El-Khawaga and Mansour, (2014); Madany,(2017) emphasized the beneficial effects of glutathione on growth, nutritional status, yield and fruit quality of fruit crops.

Previous studies showed that spraying antioxidants on citrus was responsible for reducing abiotic stresses and improving growth characters, yield as well as physical and chemical properties of the fruit of Balady mandarin (Hegab, 2000); Washington Navel orange trees (Abd El-Wahab, 1999; Erner et al., 1999; Ragab, 2002; Gobara, 2004; Gamal, 2006; Habasy et al., 2015); Valencia oranges (Sayed et al., 2004) and Balady orange (Abo El-Komsan et al., 2003).

Moreover, this study was designed to highlight the impact of the antioxidants namely glutathione, ascorbic acid and citric acid at various concentrations on growth, nutritional status, yield and fruit quality of Valencia orange trees growing under Beni-Suef region conditions.

\section{Materials and Methods}

This investigation was conducted during the two consecutive seasons of 2018 and 2019 on 15years- old Valencia orange trees, budded on sour orange rootstock, grown in clay loam soil Table (1) in a private orchard located at Bani Suef district, Bani Suef Governorate, Egypt; planted at 5x5 meters apart and irrigated under flood irrigation system.

Table 1: Orchard soil Analysis:

\begin{tabular}{ll}
\hline Characters & Values \\
\hline Sand \% & 11.5 \\
Silt \% & 10.5 \\
Clay \% & 78.0 \\
Texture & Clay -loam \\
pH (1:2.5 extract) & 7.8 \\
E.C. (1:2.5 extract) (ppm) & 1.98 \\
Organic matter \% & 1.9 \\
Total CaCO3 \% & 2.22 \\
N \% & 0.1 \\
P (ppm) & 4.8 \\
K (ammonium acetate) (ppm) & 485.0 \\
Fe (ppm) & 3.1 \\
Mn (ppm) & 2.8 \\
Zn (ppm) & 3.9 \\
\hline
\end{tabular}

This research included the following thirteen treatments, as single or combined foliar applications of (glutathione, ascorbic acid and citric acid) antioxidants:

1- Control (spraying with water)...... Cont.

2- Glutathione at $250 \mathrm{ppm} . . . . . \ldots \ldots \ldots . . . \mathrm{G} 1$

3- Glutathione at $500 \mathrm{ppm}$............ G2

4- Glutathione at $1000 \mathrm{ppm} \mathrm{..............G3}$

5- Ascorbic acid at $250 \mathrm{ppm} . . . . . . . . . . . . \mathrm{A} 1$

6- Ascorbic acid at $500 \mathrm{ppm} . . . \ldots \ldots \ldots . . . \mathrm{A} 2$ 
7- Ascorbic acid at $1000 \mathrm{ppm} . . . \ldots \ldots \ldots$..... 3

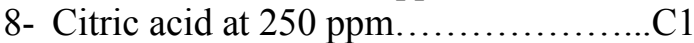

9- Citric acid at $500 \mathrm{ppm}$.................C2

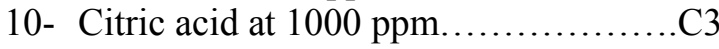

11- $\mathrm{G} 1+\mathrm{A} 1+\mathrm{C} 1$.

$12-\mathrm{G} 2+\mathrm{A} 2+\mathrm{C} 2$.

13- $\mathrm{G} 3+\mathrm{A} 3+\mathrm{C} 3$.

Each treatment was replicated three times, as one tree per each. Treatments were sprayed at three various concentrations; 250, 500 and 1000 ppm each at three times; just after fruit setting (the $1^{\text {st }}$ week of May) and replicated after two months intervals (at the $1^{\text {st }}$ week of both July and September). Triton $\mathrm{B}$ as a wetting agent was applied at $0.5 \mathrm{~cm} / \mathrm{L}$. for all a spraying solutions were applied till run off (about $10 \mathrm{~L} /$ tree). The control treatment received the tap water only and triton B. The experiment was laid out in randomized complete block design (RCBD) with three replications in both seasons.

\subsection{Experimental parameters}

The following measurements were studied as follow:

\subsection{Vegetative growth characters}

Ten flushing of the previous spring cycle (1-year- old) were labeled for each tree. Average shoot length and thickness were measured.

At the mid of September 20 leaves were picked from non- fruiting shoots for measuring leaf area $(\mathrm{cm})^{2}$ using the following equation as reported by Ahmed and Morsy, (1999).

Leaf area $=0.49(\mathrm{~L} \mathrm{x} \mathrm{W})+19.09=(\mathrm{cm})^{2}$

Where $\mathrm{L}=$ maximum length, $\mathrm{W}=$ maximum width $(\mathrm{cm})$.

\subsection{Leaf chemical composition}

At the mid of September: samples of full expanded leaves were taken from spring flushed tagged shoots during both seasons and used for determination of the following chemicals:

a) Plant pigments (mg/g F.W.): Chlorophyll a \& b and total carotenoids were determined according to (Von-Wettstein,(1957); Hiscox and Isralstam,(1979), and calculated as follow:

Chl.a $=(9.784 \times \mathrm{E} 662)-(0.99 \times \mathrm{E} 664)=\mathrm{mg} / \mathrm{ml}$

Chl.b $=(21.426 \times \mathrm{E} 644)-(4.65 \times \mathrm{E} 662)=\mathrm{mg} / \mathrm{ml}$

Total carotenoids $=(4.965 \times \mathrm{E} 440)-0.268(\mathrm{Chl} . \mathrm{a}+\mathrm{Chl} . \mathrm{b})$.

b) Total carbohydrates (\%): It was determined colorimetrically at 490 -mu wave length, using the phenol sulfuric acid method described by Smith et al., (1956).

c) Leaf mineral contents: macro-elements $\mathrm{N}, \mathrm{P}, \mathrm{K}$ and $\mathrm{Mg}$ were determined as (\%) and micro-elements $\mathrm{Zn}, \mathrm{Fe}$ and Mn were determined as (ppm) according to (Summer,(1985); Wilde et al. (1985).

\subsection{Fruit set, yield and fruit quality}

a) Initial fruit set: Four shoots (about $0.5 \mathrm{inch}$ ) at the cardinal point were labeled per each tree to determine the number of flowers and initial fruit set $\%$ as follows:

$$
\text { Initial fruit set } \%=\frac{\text { No. of fruitlets }}{\text { No. of flowers }} \times 100
$$

b) Tree Yield: After maturity stage (mid of September) the average number of fruits / tree was counted in both seasons. Moreover, at harvest stage, 20 fruits for each replicate were picked and weighed, then the tree yield was theoretically calculated $(\mathrm{kg})$.

c) Fruit quality:

For each season, at harvest stage , 20 fruits / tree were picked and prepared for the determination of the following physical and chemical properties:. 


\section{i) Fruit physical characters:}

Fruit dimensions i.e. height and diameter $(\mathrm{cm})$ and fruit shape were determined, juice $(\%)$ and fruit peel weight $(\mathrm{g})$ were measured.

\section{ii) Fruit chemical characters:}

T.S.S. \%, total acidity \% (as citric acid/ $100 \mathrm{ml}$ juice) and vitamin C content as $\mathrm{mg} / 100 \mathrm{ml}$ juice were determined as described in A.O.A.C., (2000) and the reducing and nonreducing sugars \% were determined as the methods of Lane and Eynon, (1965); A.O.A.C., (2000).

\subsection{Statistical analysis:}

As the New L.S.D. at 5\% level for making all comparisons among the treatments (Mead et al., (1993); Duncan (1955) for determine the significance variations were used.

\section{Results}

\subsection{Vegetative growth characters}

Results presented in Table (2) showed that spraying glutathione, ascorbic acid and citric acid either alone or in combinations enhanced significantly vegetative growth traits i.e. shoot length, thickness and leaf area in compared to the control. Glutathione was more effective than the other two compounds. The stimulation of growth was proportional to increase in the concentration. Whereas, higher significant values were recorded when the trees treated with the three compounds at higher concentrations. These results were true during 2018 and 2019 seasons.

Table 2: Effect of some antioxidants on Valencia orange trees vegetative growth during (2018\&2019) seasons.

\begin{tabular}{ccccccc}
\hline \multirow{2}{*}{ Treatments } & \multicolumn{2}{c}{ Shoot length $\mathbf{( c m )}$} & \multicolumn{2}{c}{ Shoot thickness (mm) } & \multicolumn{2}{c}{ Leaf area (cm) } \\
\cline { 2 - 6 } & $\mathbf{2 0 1 8}$ & $\mathbf{2 0 1 9}$ & $\mathbf{2 0 1 8}$ & $\mathbf{2 0 1 9}$ & $\mathbf{2 0 1 8}$ & $\mathbf{2 0 1 9}$ \\
\hline Cont. & $4.2 \mathrm{f}$ & $4.1 \mathrm{~h}$ & $1.5 \mathrm{k}$ & $1.4 \mathrm{j}$ & $15.6 \mathrm{~h}$ & $15.8 \mathrm{i}$ \\
G1 & $5.9 \mathrm{~b}$ & $6.1 \mathrm{bc}$ & $2.7 \mathrm{ef}$ & $2.8 \mathrm{de}$ & $19.2 \mathrm{~d}$ & $19.4 \mathrm{de}$ \\
G2 & $6.1 \mathrm{~b}$ & $6.4 \mathrm{~b}$ & $2.9 \mathrm{de}$ & $3.0 \mathrm{c} \mathrm{d}$ & $19.6 \mathrm{~d}$ & $19.9 \mathrm{~d}$ \\
G3 & $6.3 \mathrm{a}$ & $6.6 \mathrm{a}$ & $3.1 \mathrm{~cd}$ & $3.2 \mathrm{~b} \mathrm{c}$ & $20.8 \mathrm{c}$ & $21.0 \mathrm{c}$ \\
A1 & $5.2 \mathrm{~d}$ & $5.4 \mathrm{e}$ & $2.2 \mathrm{hi}$ & $2.3 \mathrm{~g}$ & $18.2 \mathrm{e}$ & $18.4 \mathrm{ef}$ \\
A2 & $5.4 \mathrm{c}$ & $5.7 \mathrm{de}$ & $2.4 \mathrm{gh}$ & $2.5 \mathrm{f}$ & $18.6 \mathrm{de}$ & $18.7 \mathrm{e}$ \\
A3 & $5.5 \mathrm{c}$ & $5.8 \mathrm{c} \mathrm{d}$ & $2.5 \mathrm{fg}$ & $2.6 \mathrm{ef}$ & $18.8 \mathrm{de}$ & $18.9 \mathrm{e}$ \\
C1 & $4.3 \mathrm{f}$ & $4.5 \mathrm{~g}$ & $1.8 \mathrm{j}$ & $1.8 \mathrm{i}$ & $16.6 \mathrm{~g}$ & $16.8 \mathrm{~h}$ \\
C2 & $4.8 \mathrm{e}$ & $4.9 \mathrm{f}$ & $1.9 \mathrm{j}$ & $2.0 \mathrm{~h} \mathrm{i}$ & $17.3 \mathrm{fg}$ & $17.4 \mathrm{gh}$ \\
C3 & $4.9 \mathrm{e}$ & $5.0 \mathrm{f}$ & $2.0 \mathrm{i} \mathrm{j}$ & $2.1 \mathrm{~g} \mathrm{~h}$ & $17.8 \mathrm{f}$ & $17.9 \mathrm{fg}$ \\
G1+A1+C1 & $5.6 \mathrm{c}$ & $6.8 \mathrm{a}$ & $3.3 \mathrm{bc}$ & $3.4 \mathrm{a} \mathrm{b}$ & $21.9 \mathrm{~b}$ & $22.2 \mathrm{~b}$ \\
G2+A2+C2 & $6.3 \mathrm{a}$ & $6.4 \mathrm{~b}$ & $3.5 \mathrm{ab}$ & $3.6 \mathrm{a}$ & $22.6 \mathrm{~b}$ & $22.9 \mathrm{~b}$ \\
G3+A3+C3 & $6.4 \mathrm{a}$ & $6.6 \mathrm{a}$ & $3.6 \mathrm{a}$ & $3.6 \mathrm{a}$ & $23.4 \mathrm{a}$ & $24.0 \mathrm{a}$ \\
\hline New L.S.D. at 5\% & 0.2 & 0.3 & 0.2 & 0.2 & 0.7 & 0.8 \\
\hline G= Glutathione; A= Ascorbic acid; C=Citric acid & & &
\end{tabular}

\subsection{Leaf chemical composition}

\subsubsection{Leaf pigments and total carbohydrates:}

As shown in Table (3), it is obvious that combined applications of glutathione, ascorbic acid and citric acid each at high concentration, $(100 \mathrm{ppm})$ significantly increased leaf content of pigments namely chlorophyll a \& b ; total chlorophyll., and carotenoids , as well as total carbohydrates when compared to the individual ones and the control. Similar results were announced during (2018 and 2019) seasons.

\subsubsection{Leaf mineral contents}

Results presented in Table (4) revealed that spraying glutathione, ascorbic acid and citric acid in combinations significantly gave the highest leaf mineral contents i.e. $\mathrm{N}, \mathrm{P}, \mathrm{K}$ and $\mathrm{Mg}$. also, $\mathrm{Zn}, \mathrm{Mn}$ and Fe as compared to the control. Higher significant values were obtained by foliar spray with the three compounds athigher concentrations. These results were true during (2018 and 019) seasons. 
Table 3: Effect of some antioxidants on Valencia orange leaf pigments and carbohydrates contents during (2018 \& 2019) seasons.

\begin{tabular}{|c|c|c|c|c|c|c|c|c|c|c|}
\hline \multirow[t]{2}{*}{ Treatments } & \multicolumn{2}{|c|}{$\begin{array}{c}\text { Chl. a } \\
\text { (mg/ } 1 \text { g. fw.) }\end{array}$} & \multicolumn{2}{|c|}{$\begin{array}{c}\text { Chl. b } \\
\text { (mg/ } 1 \text { g. fw.) }\end{array}$} & \multicolumn{2}{|c|}{$\begin{array}{c}\text { Tot. chls } \\
\text { (mg/ } 1 \text { g. fw.) }\end{array}$} & \multicolumn{2}{|c|}{$\begin{array}{c}\text { Total } \\
\text { carotenoids } \\
\text { (mg/ } 1 \text { g. fw.) } \\
\end{array}$} & \multicolumn{2}{|c|}{$\begin{array}{c}\text { Total } \\
\text { Carbohydrates } \\
(\%)\end{array}$} \\
\hline & 2018 & 2019 & 2018 & 2019 & 2018 & 2019 & 2018 & 2019 & 2018 & 2019 \\
\hline Cont. & $2.3 \mathrm{i}$ & $2.4 \mathrm{i}$ & $1.1 \mathrm{i}$ & $1.1 \mathrm{e}$ & $3.4 \mathrm{j}$ & $3.5 \mathrm{j}$ & $1.0 \mathrm{i}$ & $1.0 \mathrm{~h}$ & $14.0 \mathrm{~g}$ & $14.0 \mathrm{j}$ \\
\hline G1 & $4.5 \mathrm{e}$ & $4.6 \mathrm{f}$ & $2.6 \mathrm{e}$ & $2.7 \mathrm{c}$ & $7.1 \mathrm{e}$ & $7.3 \mathrm{e}$ & $3.0 \mathrm{~d}$ & $3.3 \mathrm{c}$ & $17.2 \mathrm{~cd}$ & $17.3 \mathrm{def}$ \\
\hline G2 & $4.9 \mathrm{~d}$ & $5.2 \mathrm{e}$ & $2.9 \mathrm{~d}$ & $3.2 \mathrm{~b}$ & $7.8 \mathrm{~d}$ & $8.4 \mathrm{~d}$ & $3.3 \mathrm{c}$ & $3.5 \mathrm{bc}$ & $17.8 \mathrm{bc}$ & $17.9 \mathrm{~cd}$ \\
\hline G3 & $5.5 \mathrm{c}$ & $5.8 \mathrm{~d}$ & $3.2 \mathrm{c}$ & $3.4 \mathrm{~b}$ & $8.7 \mathrm{c}$ & $9.2 \mathrm{c}$ & $3.4 \mathrm{bc}$ & $3.6 \mathrm{bc}$ & $18.1 \mathrm{~b}$ & $18.2 \mathrm{c}$ \\
\hline A1 & 3.8 & $3.9 \mathrm{~g}$ & $1.8 \mathrm{~g}$ & $1.9 \mathrm{~d}$ & $5.6 \mathrm{~g}$ & $5.8 \mathrm{~g}$ & $2.2 \mathrm{f}$ & $2.4 \mathrm{e}$ & $16.1 \mathrm{e}$ & $16.3 \mathrm{~g}$ \\
\hline $\mathbf{A} 2$ & $3.9 \mathrm{f}$ & $4.1 \mathrm{~g}$ & $2.2 \mathrm{f}$ & $2.4 \mathrm{c}$ & $6.1 \mathrm{f}$ & $6.5 \mathrm{f}$ & $2.4 \mathrm{ef}$ & $2.8 \mathrm{~d}$ & $16.6 \mathrm{de}$ & $16.8 \mathrm{fg}$ \\
\hline A3 & $4.4 \mathrm{e}$ & $4.5 \mathrm{f}$ & $2.5 \mathrm{e}$ & $2.6 \mathrm{c}$ & $6.9 \mathrm{e}$ & $7.1 \mathrm{e}$ & $2.5 \mathrm{e}$ & $2.9 \mathrm{~d}$ & $17.0 \mathrm{~d}$ & $17.2 \mathrm{ef}$ \\
\hline $\mathrm{C} 1$ & $2.8 \mathrm{~h}$ & $2.9 \mathrm{~h}$ & $1.2 \mathrm{i}$ & $1.3 \mathrm{e}$ & $4.0 \mathrm{i}$ & $4.2 \mathrm{i}$ & $1.4 \mathrm{~h}$ & $1.5 \mathrm{~g}$ & $14.8 \mathrm{f}$ & $14.9 \mathrm{i}$ \\
\hline $\mathrm{C2}$ & $3.3 \mathrm{~g}$ & $3.4 \mathrm{~h}$ & $1.5 \mathrm{~h}$ & $1.7 \mathrm{~d}$ & $4.8 \mathrm{~h}$ & $5.1 \mathrm{~h}$ & $1.8 \mathrm{~g}$ & $1.9 \mathrm{f}$ & $15.3 \mathrm{f}$ & $15.4 \mathrm{~h} \mathrm{i}$ \\
\hline C3 & $3.7 \mathrm{f}$ & $3.8 \mathrm{~g}$ & $1.7 \mathrm{gh}$ & $1.9 \mathrm{~d}$ & $5.4 \mathrm{~g}$ & 5.7 & $1.9 \mathrm{~g}$ & $2.0 \mathrm{f}$ & $15.8 \mathrm{e}$ & $15.9 \mathrm{gh}$ \\
\hline $\mathrm{G} 1+\mathrm{A} 1+\mathrm{C} 1$ & $5.7 \mathrm{c}$ & $5.9 \mathrm{c}$ & $3.3 \mathrm{c}$ & $3.5 \mathrm{~b}$ & $9.0 \mathrm{c}$ & $9.4 \mathrm{c}$ & $3.6 \mathrm{~b}$ & $3.7 \mathrm{~b}$ & $18.2 \mathrm{~b}$ & $18.3 \mathrm{bc}$ \\
\hline $\mathrm{G} 2+\mathrm{A} 2+\mathrm{C} 2$ & $6.6 \mathrm{~b}$ & $6.9 \mathrm{~b}$ & $3.8 \mathrm{~b}$ & $4.0 \mathrm{a}$ & $10.4 \mathrm{~b}$ & $10.9 \mathrm{~b}$ & $3.9 \mathrm{a}$ & $4.0 \mathrm{a}$ & $18.8 \mathrm{ab}$ & $18.9 \mathrm{ab}$ \\
\hline $\mathbf{G} 3+\mathbf{A} 3+\mathrm{C} 3$ & $7.2 \mathrm{a}$ & $7.4 \mathrm{a}$ & $4.1 \mathrm{a}$ & $4.3 \mathrm{a}$ & $11.3 \mathrm{a}$ & $11.7 \mathrm{a}$ & $4.0 \mathrm{a}$ & $4.1 \mathrm{a}$ & $19.0 \mathrm{a}$ & $19.1 \mathrm{a}$ \\
\hline $\begin{array}{c}\text { New LSD } \\
\text { at } 5 \%\end{array}$ & 0.2 & 0.3 & 0.2 & 0.3 & 0.4 & 0.5 & 0.2 & 0.3 & 0.6 & 0.6 \\
\hline
\end{tabular}

Table 4: Effect of some antioxidants on Valencia orange leaf elements content during (2018 \& 2019) seasons.

\begin{tabular}{ccccccccc}
\hline \multirow{2}{*}{ Treatments } & \multicolumn{2}{c}{ Leaf N \% } & \multicolumn{2}{c}{ Leaf P \% } & \multicolumn{2}{c}{ Leaf K \% } & \multicolumn{2}{c}{ Leaf Mg \% } \\
\cline { 2 - 9 } & $\mathbf{2 0 1 8}$ & $\mathbf{2 0 1 9}$ & $\mathbf{2 0 1 8}$ & $\mathbf{2 0 1 9}$ & $\mathbf{2 0 1 8}$ & $\mathbf{2 0 1 9}$ & $\mathbf{2 0 1 8}$ & $\mathbf{2 0 1 9}$ \\
\hline Cont. & $1.60 \mathrm{~h}$ & $1.61 \mathrm{f}$ & $0.16 \mathrm{~d}$ & $0.16 \mathrm{c}$ & $1.10 \mathrm{j}$ & $1.10 \mathrm{~h}$ & $0.61 \mathrm{~h}$ & $0.62 \mathrm{~g}$ \\
G1 & $1.91 \mathrm{~d}$ & $1.91 \mathrm{c}$ & $0.20 \mathrm{bc}$ & $0.20 \mathrm{ab}$ & $1.33 \mathrm{f}$ & $1.37 \mathrm{de}$ & $0.80 \mathrm{~d}$ & $0.81 \mathrm{~d}$ \\
G2 & $1.98 \mathrm{c}$ & $1.99 \mathrm{~b}$ & $0.20 \mathrm{bcd}$ & $0.20 \mathrm{ab}$ & $1.37 \mathrm{e}$ & $1.40 \mathrm{~d}$ & $0.90 \mathrm{c}$ & $0.92 \mathrm{~cd}$ \\
G3 & $2.00 \mathrm{c}$ & $2.01 \mathrm{~b}$ & $0.21 \mathrm{bc}$ & $0.22 \mathrm{a}$ & $1.44 \mathrm{~d}$ & $1.48 \mathrm{c}$ & $0.95 \mathrm{~b}$ & $0.97 \mathrm{bc}$ \\
$\mathbf{A 1}$ & $1.81 \mathrm{f}$ & $1.82 \mathrm{~d}$ & $0.18 \mathrm{~cd}$ & $0.12 \mathrm{~d}$ & $1.25 \mathrm{~h}$ & $1.26 \mathrm{f}$ & $0.74 \mathrm{edf}$ & $0.76 \mathrm{e}$ \\
$\mathbf{A 2}$ & $1.86 \mathrm{e}$ & $1.87 \mathrm{c}$ & $0.19 \mathrm{bcd}$ & $0.19 \mathrm{ab}$ & $1.28 \mathrm{gh}$ & $1.29 \mathrm{f}$ & $0.77 \mathrm{de}$ & $0.78 \mathrm{de}$ \\
$\mathbf{A 3}$ & $1.90 \mathrm{~d}$ & $1.91 \mathrm{c}$ & $0.19 \mathrm{bcd}$ & $0.19 \mathrm{ab}$ & $1.31 \mathrm{~g}$ & $1.34 \mathrm{ef}$ & $0.78 \mathrm{~d}$ & $0.79 \mathrm{de}$ \\
C1 & $1.68 \mathrm{~g}$ & $1.70 \mathrm{e}$ & $0.17 \mathrm{~d}$ & $0.17 \mathrm{~b}$ & $1.14 \mathrm{j}$ & $1.16 \mathrm{~g}$ & $0.66 \mathrm{~g}$ & $0.68 \mathrm{f}$ \\
$\mathbf{C 2}$ & $1.77 \mathrm{f}$ & $1.78 \mathrm{~d}$ & $0.17 \mathrm{~d}$ & $0.18 \mathrm{~b}$ & $1.19 \mathrm{i}$ & $1.21 \mathrm{f}$ & $0.71 \mathrm{f}$ & $0.74 \mathrm{e}$ \\
C3 & $1.80 \mathrm{f}$ & $1.81 \mathrm{~d}$ & $0.18 \mathrm{~cd}$ & $0.18 \mathrm{~b}$ & $1.23 \mathrm{~h}$ & $1.25 \mathrm{f}$ & $0.73 \mathrm{ef}$ & $0.76 \mathrm{e}$ \\
G1+A1+C1 & $2.01 \mathrm{c}$ & $2.03 \mathrm{~b}$ & $0.21 \mathrm{bc}$ & $0.21 \mathrm{a}$ & $1.49 \mathrm{c}$ & $1.55 \mathrm{~b}$ & $0.99 \mathrm{ab}$ & $1.02 \mathrm{~b}$ \\
G2+A2+C2 & $2.11 \mathrm{~b}$ & $2.14 \mathrm{a}$ & $0.22 \mathrm{~b}$ & $0.23 \mathrm{a}$ & $1.61 \mathrm{~b}$ & $1.69 \mathrm{a}$ & $1.11 \mathrm{a}$ & $1.13 \mathrm{a}$ \\
G3+A3+C3 & $2.16 \mathrm{a}$ & $2.19 \mathrm{a}$ & $0.27 \mathrm{a}$ & $0.23 \mathrm{a}$ & $1.71 \mathrm{a}$ & $1.73 \mathrm{a}$ & $1.12 \mathrm{a}$ & $1.14 \mathrm{a}$ \\
\hline New LSD at 5\% & 0.04 & 0.06 & 0.03 & 0.03 & 0.04 & 0.05 & 0.04 & 0.05 \\
\hline
\end{tabular}

Table 4:cont.

\begin{tabular}{ccccccc}
\hline \multirow{2}{*}{ Treatments } & \multicolumn{2}{c}{ Leaf Zn (ppm) } & \multicolumn{2}{c}{ Leaf Mn (ppm) } & \multicolumn{2}{c}{ Leaf Fe (ppm) } \\
\cline { 2 - 7 } & $\mathbf{2 0 1 8}$ & $\mathbf{2 0 1 9}$ & $\mathbf{2 0 1 8}$ & $\mathbf{2 0 1 9}$ & $\mathbf{2 0 1 8}$ & $\mathbf{2 0 1 9}$ \\
\hline Cont. & $50.8 \mathrm{i}$ & $50.7 \mathrm{i}$ & $55.0 \mathrm{i}$ & $56.0 \mathrm{i}$ & $59.0 \mathrm{~g}$ & $59.1 \mathrm{j}$ \\
G1 & $55.9 \mathrm{def}$ & $56.2 \mathrm{ef}$ & $70.1 \mathrm{~d}$ & $70.8 \mathrm{~d}$ & $72.5 \mathrm{~d}$ & $73.8 \mathrm{e}$ \\
G2 & $56.8 \mathrm{cde}$ & $57.4 \mathrm{de}$ & $73.2 \mathrm{c}$ & $73.9 \mathrm{c}$ & $76.2 \mathrm{c}$ & $77.9 \mathrm{~d}$ \\
G3 & $57.4 \mathrm{~cd}$ & $58.5 \mathrm{~cd}$ & $74.5 \mathrm{c}$ & $75.2 \mathrm{c}$ & $77.9 \mathrm{c}$ & $79.0 \mathrm{~cd}$ \\
A1 & $54.0 \mathrm{fgh}$ & $54.6 \mathrm{fg}$ & $64.2 \mathrm{f}$ & $65.8 \mathrm{~g}$ & $67.0 \mathrm{ef}$ & $67.9 \mathrm{~g}$ \\
$\mathbf{A 2}$ & $55.1 \mathrm{efg}$ & $55.6 \mathrm{efg}$ & $67.8 \mathrm{e}$ & $68.8 \mathrm{f}$ & $69.0 \mathrm{e}$ & $69.9 \mathrm{fg}$ \\
$\mathbf{A 3}$ & $55.7 \mathrm{defg}$ & $56.1 \mathrm{ef}$ & $68.2 \mathrm{e}$ & $69.2 \mathrm{e}$ & $71.3 \mathrm{~d}$ & $72.6 \mathrm{ef}$ \\
$\mathbf{C 1}$ & $52.4 \mathrm{hi}$ & $52.9 \mathrm{~h}$ & $58.2 \mathrm{~h}$ & 59.0 & $61.0 \mathrm{~h}$ & $61.6 \mathrm{i}$ \\
C2 & $53.6 \mathrm{ghi}$ & $53.8 \mathrm{~g}$ & $61.3 \mathrm{~g}$ & $62.0 \mathrm{~h}$ & $64.0 \mathrm{~g}$ & $65.5 \mathrm{~h}$ \\
C3 & $53.9 \mathrm{fgh}$ & $54.3 \mathrm{~g}$ & $61.4 \mathrm{~g}$ & $62.1 \mathrm{~h}$ & $66.0 \mathrm{fg}$ & $67.7 \mathrm{~g}$ \\
G1+A1+C1 & $58.8 \mathrm{bc}$ & $59.9 \mathrm{bc}$ & $77.1 \mathrm{~b}$ & $77.7 \mathrm{~b}$ & $79.2 \mathrm{~b}$ & $80.1 \mathrm{bc}$ \\
G2+A2+C2 & $60.2 \mathrm{ab}$ & $61.3 \mathrm{ab}$ & $81.2 \mathrm{a}$ & $82.1 \mathrm{a}$ & $83.5 \mathrm{a}$ & $84.4 \mathrm{a}$ \\
G3+A3+C3 & $61.1 \mathrm{a}$ & $62.8 \mathrm{a}$ & $81.9 \mathrm{a}$ & $82.8 \mathrm{a}$ & $84.6 \mathrm{a}$ & $85.5 \mathrm{a}$ \\
\hline New LSD at 5\% & 2.1 & 1.8 & 1.9 & 1.3 & 2.0 & 2.0 \\
\hline
\end{tabular}




\subsubsection{Fruit set, yield and its components}

As shown in Tables (5), it is obvious that initial fruit set (\%), yield and its components i.e. number of fruits / tree and fruit weight were significantly increased in response to combined applications of glutathione, ascorbic acid and citric acid rather than the single applications and control treatments. The increase was significantly associated with increasing concentrations of each compound. Higher fruit set and yield was recorded when the trees treated with glutathione, ascorbic acid and citric acid at high concentration $(1000 \mathrm{ppm})$, under such promise treatment yield per tree reached 80.0 and $82.8 \mathrm{~kg}$ during both seasons, respectively, While, the control treatment produced 48.0 and $49.4 \mathrm{~kg} /$ tree respectively, this increment was 66.7 and $67.6 \%$ over control for both seasons.

Table 5: Effect of some antioxidants on Valencia orange trees initial fruit set, yield and fruit weight during (2018 \&2019) seasons.

\begin{tabular}{ccccccccc}
\hline \multirow{2}{*}{ Treatments } & \multicolumn{2}{c}{ Initial fruit set \% } & \multicolumn{2}{c}{ No. of fruits / tree } & \multicolumn{2}{c}{ Yield/ tree (kg) } & \multicolumn{2}{c}{ Fruit weight (g) } \\
\cline { 2 - 9 } & $\mathbf{2 0 1 8}$ & $\mathbf{2 0 1 9}$ & $\mathbf{2 0 1 8}$ & $\mathbf{2 0 1 9}$ & $\mathbf{2 0 1 8}$ & $\mathbf{2 0 1 9}$ & $\mathbf{2 0 1 8}$ & $\mathbf{2 0 1 9}$ \\
\hline Cont. & $11.1 \mathrm{~h}$ & $11.2 \mathrm{f}$ & $300.0 \mathrm{j}$ & $305.0 \mathrm{i}$ & $48.0 \mathrm{~m}$ & 49.41 & $160.0 \mathrm{~g}$ & $162.0 \mathrm{i}$ \\
G1 & $14.4 \mathrm{~d}$ & $14.6 \mathrm{c}$ & $360.0 \mathrm{e}$ & $365.0 \mathrm{~d}$ & $64.8 \mathrm{f}$ & $65.7 \mathrm{f}$ & $180.0 \mathrm{~d}$ & $180.0 \mathrm{ef}$ \\
G2 & $15.8 \mathrm{c}$ & $16.1 \mathrm{~b}$ & $377.0 \mathrm{~d}$ & $380.0 \mathrm{c}$ & $71.6 \mathrm{e}$ & $72.6 \mathrm{e}$ & $190.0 \mathrm{c}$ & $191.0 \mathrm{~d}$ \\
G3 & $16.1 \mathrm{bc}$ & $16.3 \mathrm{~b}$ & $380.0 \mathrm{~cd}$ & $382.0 \mathrm{c}$ & $72.6 \mathrm{~d}$ & $73.7 \mathrm{~d}$ & $191.0 \mathrm{bc}$ & $193.0 \mathrm{~cd}$ \\
$\mathbf{A 1}$ & $12.9 \mathrm{e}$ & $13.0 \mathrm{~d}$ & $340.0 \mathrm{~g}$ & $342.0 \mathrm{f}$ & $58.8 \mathrm{i}$ & $59.9 \mathrm{i}$ & $173.0 \mathrm{e}$ & $175.0 \mathrm{~g}$ \\
A2 & $14.0 \mathrm{~d}$ & $14.2 \mathrm{c}$ & $351.0 \mathrm{f}$ & $354.0 \mathrm{e}$ & $62.5 \mathrm{~h}$ & $63.4 \mathrm{~h}$ & $178.0 \mathrm{~d}$ & $179.0 \mathrm{f}$ \\
A3 & $14.3 \mathrm{~d}$ & $14.5 \mathrm{c}$ & $353.0 \mathrm{ef}$ & $356.0 \mathrm{e}$ & $63.5 \mathrm{~g}$ & $64.4 \mathrm{~g}$ & $180.0 \mathrm{~d}$ & $181.0 \mathrm{e}$ \\
C1 & $11.8 \mathrm{~g}$ & $11.9 \mathrm{e}$ & $318.0 \mathrm{i}$ & $320.0 \mathrm{~h}$ & 52.51 & $53.1 \mathrm{k}$ & $165.0 \mathrm{f}$ & $166.0 \mathrm{~h}$ \\
C2 & $12.3 \mathrm{fg}$ & $12.4 \mathrm{de}$ & $329.0 \mathrm{~h}$ & $331.0 \mathrm{~g}$ & $56.3 \mathrm{k}$ & $57.6 \mathrm{j}$ & $171.0 \mathrm{e}$ & $174.0 \mathrm{~g}$ \\
C3 & $12.5 \mathrm{ef}$ & $12.6 \mathrm{~d}$ & $333.0 \mathrm{gh}$ & $340.0 \mathrm{f}$ & $57.6 \mathrm{j}$ & $59.5 \mathrm{i}$ & $173.0 \mathrm{e}$ & $175.0 \mathrm{~g}$ \\
G1+A1+C1 & $16.5 \mathrm{~b}$ & $16.6 \mathrm{~b}$ & $388.0 \mathrm{bc}$ & $390.0 \mathrm{~b}$ & $74.9 \mathrm{c}$ & $76.1 \mathrm{c}$ & $193.0 \mathrm{~b}$ & $195.0 \mathrm{c}$ \\
G2+ A2+ C2 & $17.1 \mathrm{a}$ & $17.3 \mathrm{a}$ & $395.0 \mathrm{ab}$ & $399.0 \mathrm{a}$ & $78.6 \mathrm{~b}$ & $79.8 \mathrm{~b}$ & $199.0 \mathrm{a}$ & $200.0 \mathrm{~b}$ \\
G3+A3+C3 & $17.3 \mathrm{a}$ & $17.5 \mathrm{a}$ & $398.0 \mathrm{a}$ & $402.0 \mathrm{a}$ & $80.0 \mathrm{a}$ & $82.8 \mathrm{a}$ & $201.0 \mathrm{a}$ & $206.0 \mathrm{a}$ \\
\hline New LSD at 5\% & 0.5 & 0.6 & 8.2 & 8.4 & 0.6 & 0.7 & 2.0 & 2.6 \\
\hline
\end{tabular}

\subsection{Fruit quality attributes}

\subsubsection{Fruit physical characters}

Combined applications of glutathione, ascorbic acid and citric acid significantly increased fruit physical characters such as fruit juice $\%$ and reduced fruit peel weight relative to the other treatments and the control (Table 6). On the other hand, all treatments under study had insignificant effect on fruit shape index during the two seasons. In additions, the promotion on quality of the fruits was significantly associated with increasing concentrations of each compound. The best result with regard to fruit physical characters were obtained when the trees were treated with a mixture of glutathione, ascorbic acid and citric acid each at 1000 ppm. These results were true during 2018 and 2019 seasons.

Table 6: Effect of some antioxidants on Valencia orange fruits physical characteristics during (2018 \&2019) seasons

\begin{tabular}{ccccccc}
\hline \multirow{2}{*}{ Treatments } & \multicolumn{2}{c}{ Fruit Shape Index } & \multicolumn{2}{c}{ Juice \% } & \multicolumn{2}{c}{ Fruit peel weight (g) } \\
\cline { 2 - 7 } & $\mathbf{2 0 1 8}$ & $\mathbf{2 0 1 9}$ & $\mathbf{2 0 1 8}$ & $\mathbf{2 0 1 9}$ & $\mathbf{2 0 1 8}$ & $\mathbf{2 0 1 9}$ \\
\hline Cont. & 1.12 & 1.12 & $40.0 \mathrm{~h}$ & $40.0 \mathrm{~h}$ & $24.0 \mathrm{a}$ & $23.5 \mathrm{a}$ \\
G1 & 1.13 & 1.13 & $44.0 \mathrm{~d}$ & $44.2 \mathrm{~d}$ & $17.0 \mathrm{f}$ & $16.9 \mathrm{e}$ \\
G2 & 1.01 & 1.13 & $45.0 \mathrm{c}$ & $45.8 \mathrm{c}$ & $16.0 \mathrm{fg}$ & $15.9 \mathrm{f}$ \\
G3 & 1.13 & 1.13 & $45.7 \mathrm{c}$ & $45.9 \mathrm{c}$ & $15.9 \mathrm{~g}$ & $15.8 \mathrm{fg}$ \\
A1 & 1.14 & 1.14 & $41.9 \mathrm{f}$ & $42.1 \mathrm{f}$ & $20.0 \mathrm{~d}$ & $19.9 \mathrm{c}$ \\
A2 & 1.13 & 1.16 & $43.0 \mathrm{e}$ & $43.3 \mathrm{e}$ & $19.0 \mathrm{de}$ & $18.9 \mathrm{~d}$ \\
A3 & 1.13 & 1.13 & $43.3 \mathrm{~d}$ & $43.6 \mathrm{de}$ & $18.9 \mathrm{e}$ & $18.8 \mathrm{~d}$ \\
C1 & 1.13 & 1.13 & $40.9 \mathrm{~g}$ & $41.1 \mathrm{~g}$ & $23.0 \mathrm{ab}$ & $23.0 \mathrm{a}$ \\
C2 & 1.14 & 1.14 & $41.8 \mathrm{fg}$ & $42.0 \mathrm{f}$ & $22.0 \mathrm{~b}$ & $21.9 \mathrm{~b}$ \\
C3 & 1.15 & 1.14 & $41.9 \mathrm{f}$ & $42.1 \mathrm{f}$ & $21.9 \mathrm{c}$ & $21.8 \mathrm{~b}$ \\
G1+A1+C1 & 1.13 & 1.13 & $47.0 \mathrm{~b}$ & $47.2 \mathrm{~b}$ & $15.0 \mathrm{gh}$ & $14.9 \mathrm{~g}$ \\
G2+A2+C2 & 1.13 & 1.13 & $48.2 \mathrm{a}$ & $48.4 \mathrm{a}$ & $14.0 \mathrm{~h}$ & $13.9 \mathrm{~h}$ \\
G3+A3+C3 & 1.11 & 1.11 & $48.4 \mathrm{a}$ & $48.5 \mathrm{a}$ & $14.0 \mathrm{~h}$ & $13.8 \mathrm{~h}$ \\
\hline New LSD at 5\% & NS & NS & 0.7 & 0.6 & 1.0 & 0.8 \\
\hline
\end{tabular}




\subsubsection{Fruit chemical characters}

As shown in Tables (7), it is obvious that all fruit chemical characters were significantly affected by all foliar application of conducted antioxidants. Combined applications of glutathione, ascorbic acid and citric acid significantly improved fruit chemical characters i.e. T.S.S., total sugars, reducing sugars and vitamin $\mathrm{C}$ and reduced total acidity as compared to the control. The promotion on quality of the fruits was significantly associated with increasing concentrations of each compound. The best result with regard to fruit chemical characters was attained by trees treated with a mixture of glutathione, ascorbic acid and citric acid each at $1000 \mathrm{ppm}$. Untreated trees produced un- favourite effects on fruit quality. These results were true during 2018 and 2019 seasons.

Table 7: Effect of some antioxidants on Valencia orange fruit chemical characteristics during (2018 $\& 2019)$ seasons.

\begin{tabular}{|c|c|c|c|c|c|c|c|c|c|c|}
\hline \multirow[t]{2}{*}{ Treatments } & \multicolumn{2}{|c|}{$\begin{array}{l}\text { T.S.S } \\
(\%)\end{array}$} & \multicolumn{2}{|c|}{$\begin{array}{c}\text { Tot. acidity } \\
\text { (\%) }\end{array}$} & \multicolumn{2}{|c|}{$\begin{array}{l}\text { Tot. sugars } \\
\text { (\%) }\end{array}$} & \multicolumn{2}{|c|}{$\begin{array}{c}\text { Reducing } \\
\text { sugars }(\%)\end{array}$} & \multicolumn{2}{|c|}{$\begin{array}{c}\text { Vit. C } \\
\text { (mg/ } 100 \mathrm{ml} \text { juice) }\end{array}$} \\
\hline & 2018 & 2019 & 2018 & 2019 & 2018 & 2019 & 2018 & 2019 & 2018 & 2019 \\
\hline Control & $10.7 \mathrm{e}$ & $10.8 \mathrm{~g}$ & $1.36 \mathrm{a}$ & $1.36 \mathrm{a}$ & $7.4 \mathrm{e}$ & $7.2 \mathrm{~g}$ & $3.6 \mathrm{f}$ & $3.5 \mathrm{i}$ & $44.4 \mathrm{~g}$ & $44.8 \mathrm{i}$ \\
\hline G1 & $11.9 \mathrm{c}$ & $12.0 \mathrm{~d}$ & $1.26 \mathrm{~d}$ & $1.25 \mathrm{e}$ & $8.5 \mathrm{c}$ & $8.6 \mathrm{~d}$ & $4.2 \mathrm{~d}$ & $4.3 \mathrm{def}$ & $47.8 \mathrm{~d}$ & $48.1 \mathrm{e}$ \\
\hline G2 & $12.3 \mathrm{~b}$ & $12.4 \mathrm{c}$ & $1.23 \mathrm{e}$ & $1.22 \mathrm{f}$ & $8.8 \mathrm{~b}$ & $8.9 \mathrm{c}$ & $4.3 \mathrm{bc}$ & $4.4 \mathrm{cde}$ & $48.9 \mathrm{c}$ & $49.2 \mathrm{~d}$ \\
\hline G3 & $12.4 \mathrm{~b}$ & $12.5 \mathrm{~b}$ & $1.22 \mathrm{ef}$ & $1.21 \mathrm{f}$ & $8.9 \mathrm{~b}$ & $9.0 \mathrm{c}$ & $4.4 \mathrm{bc}$ & $4.5 \mathrm{bcd}$ & $49.3 \mathrm{c}$ & $49.6 \mathrm{~d}$ \\
\hline A1 & $11.4 \mathrm{~d}$ & $11.5 \mathrm{e}$ & $1.31 \mathrm{c}$ & $1.31 \mathrm{c}$ & $8.0 \mathrm{~d}$ & $8.1 \mathrm{e}$ & $4.0 \mathrm{~d}$ & $4.1 \mathrm{fg}$ & $46.6 \mathrm{e}$ & $47.0 \mathrm{f}$ \\
\hline A2 & $11.8 \mathrm{c}$ & $11.8 \mathrm{~d}$ & $1.28 \mathrm{~d}$ & $1.28 \mathrm{de}$ & $8.4 \mathrm{c}$ & $8.5 \mathrm{~d}$ & $4.1 \mathrm{~d}$ & $4.2 \mathrm{ef}$ & $47.5 \mathrm{~d}$ & $47.8 \mathrm{e}$ \\
\hline A3 & $11.9 \mathrm{c}$ & $12.0 \mathrm{~d}$ & $1.27 \mathrm{~d}$ & $1.26 \mathrm{e}$ & $8.5 \mathrm{c}$ & $8.6 \mathrm{~d}$ & $4.2 \mathrm{~cd}$ & $4.3 \mathrm{df}$ & $47.6 \mathrm{de}$ & $47.9 \mathrm{e}$ \\
\hline C1 & $10.9 \mathrm{e}$ & $11.1 \mathrm{f}$ & $1.34 \mathrm{ab}$ & $1.34 \mathrm{ab}$ & $7.6 \mathrm{e}$ & $7.6 \mathrm{f}$ & $3.7 \mathrm{ef}$ & $3.8 \mathrm{~h}$ & $45.5 \mathrm{f}$ & $45.8 \mathrm{~h}$ \\
\hline $\mathrm{C} 2$ & $11.3 \mathrm{~d}$ & $11.4 \mathrm{e}$ & $1.33 \mathrm{bc}$ & $1.32 \mathrm{bc}$ & $7.9 \mathrm{de}$ & $7.9 \mathrm{e}$ & $3.9 \mathrm{de}$ & $3.9 \mathrm{~g}$ & $46.2 \mathrm{e}$ & $46.4 \mathrm{~g}$ \\
\hline $\mathrm{C3}$ & $11.4 \mathrm{~d}$ & $11.5 \mathrm{e}$ & $1.31 \mathrm{c}$ & $1.31 \mathrm{c}$ & $8.0 \mathrm{~d}$ & $8.1 \mathrm{e}$ & $4.0 \mathrm{~d}$ & $4.1 \mathrm{fg}$ & 46.6de & $46.9 \mathrm{f}$ \\
\hline $\mathbf{G}+\mathbf{A} 1+\mathbf{C} 1$ & $12.6 \mathrm{~b}$ & $12.7 \mathrm{~b}$ & $1.20 \mathrm{f}$ & $1.18 \mathrm{~g}$ & $9.0 \mathrm{ab}$ & $9.1 \mathrm{bc}$ & $4.5 \mathrm{ab}$ & $4.6 \mathrm{abc}$ & $49.9 \mathrm{~b}$ & $50.0 \mathrm{c}$ \\
\hline $\mathrm{G} 2+\mathrm{A} 2+\mathrm{C} 2$ & $13.7 \mathrm{a}$ & $13.8 \mathrm{a}$ & $1.16 \mathrm{~g}$ & $1.13 \mathrm{~h}$ & $9.2 \mathrm{a}$ & $9.3 \mathrm{ab}$ & $4.6 \mathrm{ab}$ & $4.7 \mathrm{ab}$ & $50.7 \mathrm{a}$ & $50.9 \mathrm{~b}$ \\
\hline $\mathrm{G} 3+\mathrm{A3}+\mathrm{C} 3$ & $13.8 \mathrm{a}$ & $13.9 \mathrm{a}$ & $1.15 \mathrm{~g}$ & $1.14 \mathrm{~h}$ & $9.3 \mathrm{a}$ & $9.4 \mathrm{a}$ & $4.7 \mathrm{a}$ & $4.8 \mathrm{a}$ & $51.0 \mathrm{a}$ & $51.5 \mathrm{a}$ \\
\hline $\begin{array}{c}\text { New LSD at } \\
\mathbf{5 \%}\end{array}$ & 0.3 & 0.2 & 0.02 & 0.02 & 0.3 & 0.2 & 0.3 & 0.2 & 0.4 & 0.4 \\
\hline
\end{tabular}

\section{Discussion}

The higher content of glutathione from (glycine, glutamic acid and cysteine) as well as its action on enhancing sulfur metabolism and defense gene and reducing reactive oxygen species (ROS) could explain the present results (Mulleineaux and Rausch, 2005). The promoting effect of glutathione on growth and nutritional status, surely reflected on enhancing fruit set \%, yield and fruit quality.

The obtained results are nearly in agreement with those obtained by Abd El aal et al., (2012); Gad El Kareem (2012) on Taimour mango trees, El-Khawaga and Mansour (2014);Ahmed and Habasy (2017) on Washington Navel orange and Saied (2019) on Ewaise mango trees.

Ascorbic acid and citric acid with their anti-oxidative properties play a definite role in preventing the free radicals from oxidation of lipids and the loss of permedity, important role in controlling the incidence of fungal attack. Antioxidants are responsible for protecting plant cells from senescence and death and they enhance nutrients uptake by plants. These results are in agreement with those obtained by Abo El-Komsan et al., (2003); Saied (2005);Ali (2008); Abd Elaal, et al., (2012), Gamal (2013) ;Saied (2019). Finally, the positive effect of glutathione, ascorbic acid and citric acid on chemical composition may be due to that glutathione increased the availability of most nutrients.

\section{Conclusion}

Treating Valencia orange trees grown under Beni-Suef region conditions with a mixture of glutathione, ascorbic acid and citric acid each at $(1000 \mathrm{ppm})$ three times just after fruit setting (the $1^{\text {st }}$ week of May) and two months intervals (the $1^{\text {st }}$ week of July and September) gave the best results with regard to tree growth, fruit setting, yield and fruit quality. 


\section{References}

Abd El Aal, A.M.K., F.H. Abdel-Aziz, and R.S. Abdel-Gawad, 2012. Relation of fruiting in Balady Mandarin trees with spraying the two antioxidants citric and ascorbic acids, Egypt J. Agric. Res., 87(1): 267-284.

Abd Elaal, A.M.K., A.A.B. Masoud, and A.Y. Mohamed, 2012. Response of Taimour mango trees to application of the antioxidant glutathione. Menufia J. Agric. Res., (3): 303-310.

Abd El-Wahab, A.Y., 1999. Studies on the effect of foliar spraying with magnesium, boron and some vitamins on some vegetative and fruiting characters of Washington Navel orange (Citrus sinesis, Osb,) M.Sc. Thesis Fac. of Agric. Minia Univ. Egypt.

Abo El-Komsan, E.A., M.Y. Hegab, and Fouad, A. Amera, 2003. Response of Balady orange trees to application of some nutrients and citric acid, Egypt J. App. Sci. 18(3): 228-246.

Ahmed, F.F. Habasy, and E.Y. Randa 2017. Behavior of navel orange trees to application of the antioxidant glutathione J. Biol. Chem. Environ., Sci., 12(1):735-746.

Ahmed, F.F. and M.H. Morsy, 1999. A new method for measuring leaf area in different fruit species. Minia J. of Agric. Res. \& Develop. (19): 97-105.

Ali, Ragaa S.A. 2008. Effect of ascorbic and citric acids on fruiting of Balady mandarin trees. M.Sc. Thesis Fac. of Agric. Minia Univ. Egypt.

Association of Official Agricultural Chemists, 2000. Official Method of Analysis (A.P.A.C.) $15^{\text {th }}$ Ed., published by A.O.A.C. Washington, D.C. (U.S.A.) p. 490-510.

Duncan,B.D. 1955: Multiple range and multiple tests. Biometrics, h: 1-42.

El-Khawaga, A.S. and A.G.M. Mansour, 2014. Promoting productivity of Washington Navel orange trees by using some crop seed sprout extract, silicon and glutathione. Middle East Journal of Applied Science, 4(3): 779-785.

Erner, Y., A. Cohen, and H. Magen, 1999. Fertilizing for high yield of citrus. Basel Switzerland Inter Potash Instit. IPI Bull., 4 (59):10-20.

Gad El-Kareem, M.R., 2012. Improving productivity of Taimour mango trees by using glutathione, silicon and vitamin B Minia J. of Agric. Res. \& Develop. 32(7): 1105-1121.

Gamal, A.F.I., 2006. Response of Washington Navel orange trees to some antioxidants. M.Sc. Thesis Fac. of Agric. Minia Univ. Egypt.

Gamal, A.F.O., 2013. Fruiting of Washington navel orange trees in relation to application of seaweed extract, boron and citric acid Ph.D. Thesis Fac. of Agric. Minia Univ. Egypt.

Gobara, A.A., 2004. Growth and fruiting of Washington navel oranges in relation to foliar application of some antioxidants, Minia J. of Agric. Develop. 24(4):580-600.

Habasy, Randa E.Y., Helal, E.M. Mona, F.F. Ahmed, and M.A. Abd El- Rahman, 2015. Effect of spraying some nutrients and citric acid on fruiting of Washington navel orange trees. J. Bio. Chem. Environ Sci. 10(4): 13-24.

Hegab, M.Y., 2000. Response of Balady mandarin trees to application of citric and ascorbic acids in combined with iron and zinc. Egypt, J. App. Sci. 15(10): 194-208.

Hiscox, A. and B. Isralstam, 1979: Methods for the extraction of chlorophylls from leaf tissue without maceration. Can. J. Bot. 57:1332-1334.

Klessig, D.F., J. Durner, R. Noad, D.A. Navarre, D. Wondhenne, D. Kumar, J.M. Zhuu, J. Shah, S. Zhang, P. Kachroom, Y. Triaf, D. Pontier, E. Lam, and H. Silvo, 2000. Nitric oxide and salicylic acid signaling in plant defense proc. Nat. Acad. Sco. USA, 97: 8849-8855.

Lane, J.H., and L. Eynon, 1965. Determination of reducing sugars by means of Fehling's solution with methylene blue as internal indicator. J. Soc. Chem. Ind. Trans. 42:.32-36.

Madany, M.H.G., 2017. Response of Succary mango trees to foliar application of glutathione and boric acid. M.Sc. Thesis, Fac. of Agric. Minia Univ. Egypt.

Mead, R., R.N. Curnow, and A.M. Harted, 1993. Statistical Methods in Agricultural and Experimental Biology. $2^{\text {nd }}$ Ed. Hall, London, 10-20.

Mulleineaux, M. and T. Rausch, 2005. Glutathione, photosynthesis and the redox regulation of stress responsive gene expression photosynthesis Res. 47: 459-474.

Orth, A.B; Sfarra, A., R.J. Pell, and M. Tlen, 1993. Assessing the involvement of free radicals in fungicide toxicity using $\alpha$-Tocopherol analogs. Biochemistry and Physiology. 47: 134-141. 
Ragab, M.M., 2002. Effect of spraying urea, Ascorbic acid and NAA on fruiting of Washington navel orange trees (Citrus sinensis) M.Sc. Thesis in Fac. of Agric. Minia Univ. Egypt.

Rao, M.V., J.R. Koch, and K.P. Davis, 2000. Ozone: A tool for probing programmed cell death in plants. Plant Mol. Bio.44: 346-358.

Raskin, I., 1992. Role of salicylic acid in plant Ann. Rev. Physiol. Plant Mol. Bio. 43: 429-463.

Saied, H.H.M., 2005. Studies on tolerance of some mango cultivars to salinity and lime. Ph.D. Thesis Fac. of Agric. Minia Univ. Egypt.

Saied, H.H.M., 2019. Effect of spraying fish oil and glutathione on fruiting of Ewaise mango trees grown under sandy soil. Hortscience Journal of Suez Canal. Univ. 8 (91): 95-108.

Sandermann, H.Jr., D. Ernst, W. Heler, and C. Langebartels, 1998. Ozone: An abiotic elicitor of plant defense reactions. Trends Plant Sc., 3: 46-50.

Sayed, R.A., B.M. Soliman, and E.Q. Abo-El- Komsan, 2004. Effect of foliar sprays of some mineral nutrients, $\mathrm{GA}_{3}$ and or biostimulant on yield and fruit quality of Valencia orange trees grown in sandy soil, Egypt. J. App. Sci. 19(5): 222-238.

Smith, F., M.A. Gilles, J.K. Hamilton, and P.A. Gedess, 1956. Colorimetric methods for determination of sugar and related substan, Anal. Chem., 28: 350-356.

Summer, M.E. 1985. Diagnosis and recommendation Integrated System (DRIS) as a guide to orchard fertilization. Hort. Abst. 55(8):7502.

Vianello, A. and F. Marcim, 1991. Generation of superoxide anion and hydrogen peroxide at the surface if plant cells J. Bioenergetics and Biomembranes, 23(3): 409-423.

Von-Wettstein, D.V., 1957. Chlorophyll-Lethalc under submikroshopishe formilkechrel der plastiden celi, prp. Trop. Res. Amer. Soc. Hort. Sci. 20: 427-433.

Wilde, S.A., R.B. Corey, J.G. Lyer and G.K. Voigt, 1985. Soil and plant analysis for tree culture. $3^{\text {rd }}$ Ed. Oxford 81 BM Publishing Co., New Delhi, 1-218. 